\title{
Expression of surface and intracellular Toll-like receptors by mature mast cells
}

\author{
JUSTYNA AGIER, PAULINA ŻELECHOWSKA, ELŻBIETA KOZŁOWSKA, \\ EWA BRZEZINISKA-BEASZCZYK
}

Department of Experimental Immunology, Medical University of Lodz, Poland

\begin{abstract}
Nowadays, more and more data indicate that mast cells play an important role in host defense against pathogens. That is why it is essential to understand the expression of Toll-like receptors (TLRs) by mast cells, because these molecules play particularly significant role in initiation host defense against microorganisms as they recognize both wide range of microbial pathogen-associated molecular patterns (PAMPs) and various endogenous damage-associated molecular patterns (DAMPs) released in response to infection. Therefore, we examined the constitutive expression of both surface and endosomal TLRs in rat native fully mature tissue mast cells. By the use of qRT-PCR we found that these cells express mRNAs for TLR2, TLR3, TLR4, TLR5, TLR7, and TLR9. The expression of TLR3, TLR4, TLR5, TLR7, and TLR 9 transcripts were low and comparable and only the expression of TLR 2 transcript was significant. By the use of flow cytometry technique, we clearly documented that mast cells express TLR2, TLR4, and TLR5 on cell surface, while TLR3, TLR7, and TLR9 proteins are located both on the cell membrane and intracellularly. The highest expression was observed for TLR5 and the lowest for surface TLR7. These observations undoubtedly indicate that mature tissue mast cells have a broad set of TLR molecules, thus can recognize and bind bacterial, viral, and fungal PAMPs as well as various endogenous molecules generated in response to infection.
\end{abstract}

Key words: mast cells, pattern recognition receptors, Toll-like receptors, antimicrobial defense.

(Cent Eur J Immunol 2016; 41 (4): 333-338)

\section{Introduction}

Mast cells are long-lived resident tissue cells distributed throughout vascularized connective tissues and are especially numerous near surfaces exposed to the environment, including the skin, the respiratory system, the gastrointestinal and genitourinary tracts, i.e. at the portals of pathogen entry. These cells are a potent source of various biologically active mediators, such as granule-associated preformed mediators (e.g. histamine, neutral proteases, metalloproteinases, proteoglycans), de novo generated arachidonic acid metabolites (i.e. leukotrienes, prostaglandins, thromboxanes), and many cytokines and chemokines. Mast cell mediators can exert diverse regulatory/modulatory effects on surrounding cells and tissues [1-3]. Therefore, mast cells are involved in homeostasis maintenance and are important players in many physiological processes, such as wound healing, angiogenesis, regulation of vascular permeability, and tissue remodeling and repair [2, 3]. These cells are known to participate in inflammation and affect both innate and adaptive immune responses, as well [4-6]. Moreover, mast cells take part in different pathological processes, including chronic allergic disorders, autoimmune diseases, and neoplastic processes $[2,3,7]$.
Nowadays, more and more data indicate that mast cells are critical component of host defence against a variety of microorganisms, mainly bacteria [8] and viruses [9]. The role of these cells in protection against fungal infections is less understandable. Without a doubt, the strategic location of mast cells at the portals of infection allows them to establish quick contact with invading pathogens. Furthermore, mast cells express antimicrobial peptides such as cathelicidin and defensins [10]. Moreover, these cells have the ability to phagocytose and subsequently kill bacteria, via oxidative and non-oxidative systems [11, 12]. It should be highlighted that mast cells can kill bacteria independently of phagocytosis, because they form extracellular traps composed of DNA, histones, tryptase, and cathelicidin [13]. What is important, following phagocytosis these cells are capable of processing bacterial antigens for presentation through class I and II MHC molecules, which leads to the development of antimicrobial adaptive immunity $[11,14]$. Finally, mast cell-derived proinflammatory mediators, cytokines and chemokines induce the development of inflammation at the site of pathogen entry $[4,5,8]$.

Correspondence: Prof. Ewa Brzezińska-Błaszczyk, Department of Experimental Immunology, Medical University of Lodz, Pomorska 251, 92-215 Lodz, Poland, e-mail: ewab@csk.umed.lodz.pl

Submitted: 1.07.2016; Accepted: 22.09.2016 
The initial recognition of microorganisms is mediated by different specialized pattern recognition receptors (PRRs). These receptors identify a diverse set of microbial molecules, called pathogen-associated molecular patterns (PAMPs). What is more, PRRs can recognize various host-derived molecules, called damage-associated molecular patterns (DAMPs), released from infected or necrotic cells and damaged tissues. The PRR family includes Toll-like receptors (TLRs), C-type lectin-like receptors (CLRs), RIG-I-like receptors (RLRs), and NOD-like receptors (NLRs) $[15,16]$. The best characterized PRRs are TLR family members expressed in diverse body cells either on plasma membrane (TLR1, TLR2, TLR4, TLR5, TLR6, TLR10) or on membrane of endosomes (TLR3, TLR7, TLR8, TLR9). Without any doubt, out of all PRRs, members of the TLR family play particularly significant role in initiation host defence against pathogens because these receptors recognize both wide range of microbial PAMPs and various endogenous DAMPs released in response to infection [17-19]. Considering the important role of mast cells in antimicrobial protection it is essential to comprehend the expression of TLRs by these cells. Accordingly, we decided to examine the constitutive expression of both surface and endosomal TLRs in fully mature tissue mast cells.

\section{Material and methods}

\section{Reagents}

Dulbecco's Modified Eagle Medium (DMEM) was obtained from Biowest (Kansas City, MO, USA). Fetal Calf Serum (FCS), gentamicin, glutamine, Hank's Balanced Salt Solution (HBSS), and sodium bicarbonate were purchased from GIBCO (Gaithersburg, MD, USA). Phosphate Buffered Saline (PBS), Percoll, saponin, toluidine blue, trypan blue, and Tween 20 were obtained from Sigma-Aldrich (St. Louis, MO, USA). CellFIX was purchased from BD Biosciences (Benelux, NV, Belgium). The GeneMATRIX Universal RNA Purification Kit was purchased from EURx (Gdansk, Poland). Anti-TLR2, anti-TLR3, anti-TLR4, anti-TLR5, anti-TLR7, anti-TLR9 antibodies, nonspecific goat IgG (isotypic control), as well as fluorescein isothiocyanate (FITC)-conjugated polyclonal, donkey anti-goat and donkey anti-rabbit antibodies, and blocking peptides were obtained from Santa Cruz Biotechnology Inc. (Santa Cruz, CA, USA). The High-Capacity cDNA Reverse Transcription Kit, TaqMan ${ }^{\circledR}$ probes dyed FAM (rTLR2, rTLR3, rTLR4, rTLR5, rTLR7, rTLR9 and r $\beta$-actin), and TaqMan ${ }^{\circledR}$ Gene Expression Master Mix were purchased from Applied Biosystems (Foster City, CA, USA).

\section{Animals}

Female albino Wistar rats weighing 220-250 g, aged 3-4 months were used. The experimental procedures were approved by the Local Ethics Committee for Experiments on Animals of the Medical University of Lodz (the approval No. 20/ŁB 740/2015).

\section{Isolation of mast cells}

Mast cells were collected from peritoneal cavities of female Wistar rats by lavage, with $50 \mathrm{ml}$ of $1 \%$ HBSS supplemented with $0.015 \%$ sodium bicarbonate. After abdominal massage (90 s) the cell suspension was removed from the peritoneal cavity, centrifuged $\left(150 \mathrm{~g}, 5 \mathrm{~min}, 20^{\circ} \mathrm{C}\right)$ and washed twice in complete DMEM (cDMEM), containing DMEM supplemented with 10\% FCS, $10 \mu \mathrm{g} / \mathrm{ml}$ gentamicin, and $2 \mathrm{mM}$ glutamine. Isotonic $72.5 \%$ Percoll density gradient centrifugation $\left(190 \mathrm{~g}, 15 \mathrm{~min}, 20^{\circ} \mathrm{C}\right)$ was used for mast cell purification. Subsequently, isolated mast cells were centrifuged twice in cDMEM $\left(150 \mathrm{~g}, 5 \mathrm{~min}, 20^{\circ} \mathrm{C}\right)$. Purified mast cells were counted and resuspended in an adequate volume of cDMEM to obtain mast cell concentration of $1.5 \times 10^{6}$ cells $/ \mathrm{ml}$. Mast cells were prepared with purity $>98 \%$, as determined by metachromatic staining with toluidine blue. The viability of mast cells was over $98 \%$, as estimated by trypan blue exclusion assay [20].

\section{RNA isolation and qRT-PCR}

GeneMATRIX Universal RNA Purification Kit was used to isolate total RNA from native mast cells. Isolated RNA was reverse transcribed to cDNA using High Capacity cDNA Reverse Transcription Kit according to the manufacturer's protocol. qRT-PCR was used to determine constitutive TLR mRNAs expression. The expression of TLR2, TLR3, TLR4, TLR5, TLR7, TLR9 mRNAs as well as the expression of the endogenous $\beta$-actin mRNA were performed with using TaqMan ${ }^{\circledR}$ Gene Expression Master Mix. Reactions were carried out with the use TaqMan ${ }^{\circledR}$ probes and 7900HT Fast Real-Time PCR System (Applied Biosystems, Foster City, CA, USA). Values were normalized to the transcript level of the reference gene rat $\beta$-actin.

\section{Flow cytometric measurements}

For determination of TLR2, TLR3, TLR4, TLR5, TLR7, and TLR9 surface protein expression and TLR3, TLR7, and TLR9 intracellular protein expression flow cytometry technique was applied. Constitutive expression of TLRs was assessed on freshly isolated native mast cells. Mast cells were washed twice in the cDMEM after isolation and fixed with CellFIX solution overnight at $4^{\circ} \mathrm{C}$. Next, mast cells were washed twice in CDMEM, and then resuspended in $1 \times$ PBS. To verify the presence of TLR receptors intracellularly, mast cells were permeabilized with $0.1 \%$ saponin for $30 \mathrm{~min}$ at the room temperature. Non-permeabilized and permeabilized mast cells were stained for $1 \mathrm{~h}$ with appropriate primary antibodies (antibody dilution $1: 100$ ). For control, mast cells were stained with isotype antibodies. The appropriate blocking peptides were used to verify antibody specificity. Cells were then 
washed with PBS containing $0.05 \%$ Tween 20 and incubated for $1 \mathrm{~h}$ with FITC-conjugated secondary antibodies. Finally, cells were washed with PBS containing $0.05 \%$ Tween 20 and resuspended in $1 \times$ PBS. Immunofluorescence analysis of 20000 cells was performed by FACS Canto II flow cytometer (BD Biosciences, San Jose, CA, USA).

\section{Statistical analysis}

Statistical analysis included mean value and standard error of the mean (SEM).

\section{Results}

In this study, we determined the expression of TLRs in freshly isolated mature rat mast cells using qRT-PCR and flow cytometry. We first examined TLR2, TLR3, TLR4, TLR5, TLR7, and TLR9 transcript levels by qRT-PCR. We found that naive mature mast cells express mRNA for all the studied receptors (Fig. 1). It is necessary to point out that the expression of TLR3, TLR4, TLR5, TLR7, and TLR9 transcripts were low and comparable. Only the expression of TLR2 transcript was significant and up to six times higher than those. Controls without reverse transcriptase confirmed that the source of the products was indeed from mRNA, and not from contaminating genomic DNA.

Next, we conducted experiments, by means of flow cytometry, to establish whether tissue mast cells constitutively express TLR proteins. As can be seen in Figures 2A-C surface TLR2, TLR4, and TLR5 were detected on
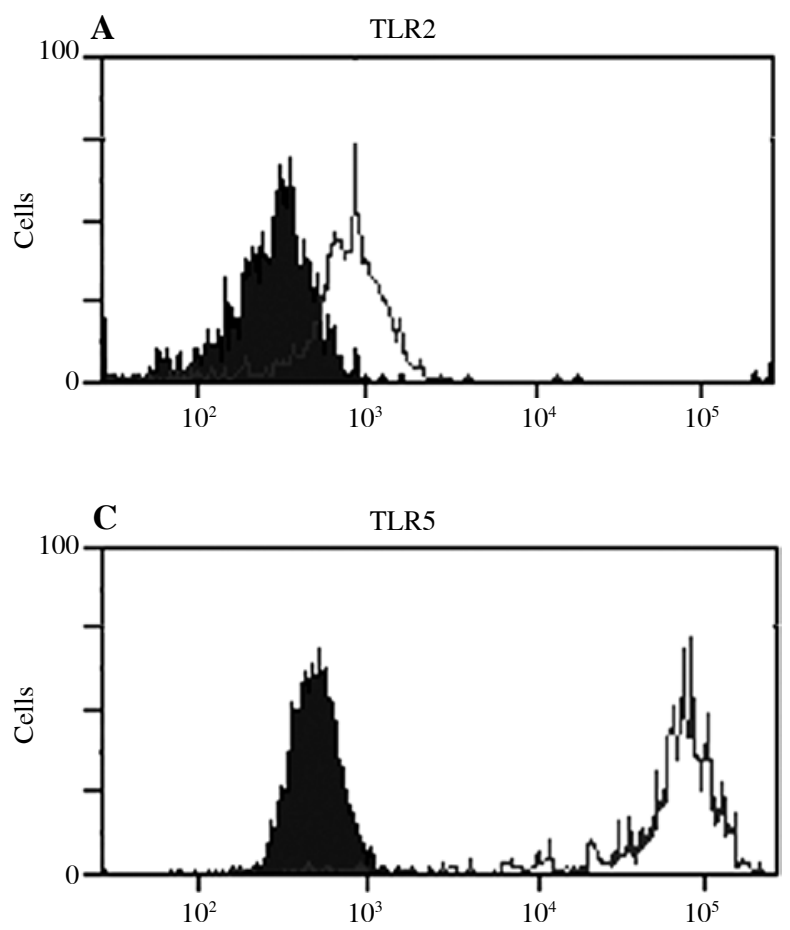

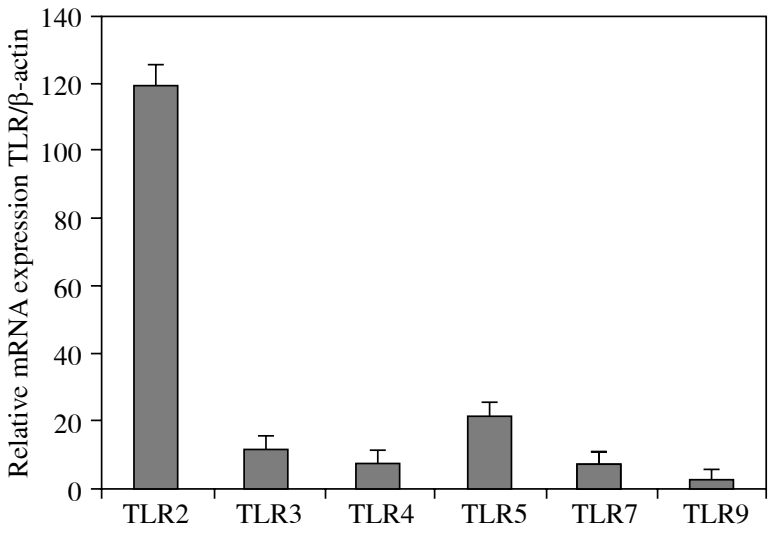

Fig 1. Constitutive mRNA expression of TLRs in freshly isolated rat peritoneal mast cells. TLR2, TLR3, TLR4, TLR5, TLR7, and TLR9 mRNA expression were examined by qRT-PCR. Relative expression levels of TLR mRNAs, normalized to housekeeping gene $\beta$-actin. Each bar represents the mean $\pm \mathrm{SEM}$ of four experiments performed

non-permeabilized native mast cells. Moreover, staining of non-permeabilized and permeabilized mast cells showed that TLR3, TLR7, and TLR9 proteins were located both on the cell surface and intracellularly (Fig. 3A-F).

A comparison of expression levels of TLRs proteins is shown in Figure 4. The highest surface protein expression was observed for TLR5 and the lowest for sTLR9. TLR5 protein expression was over four times greater than

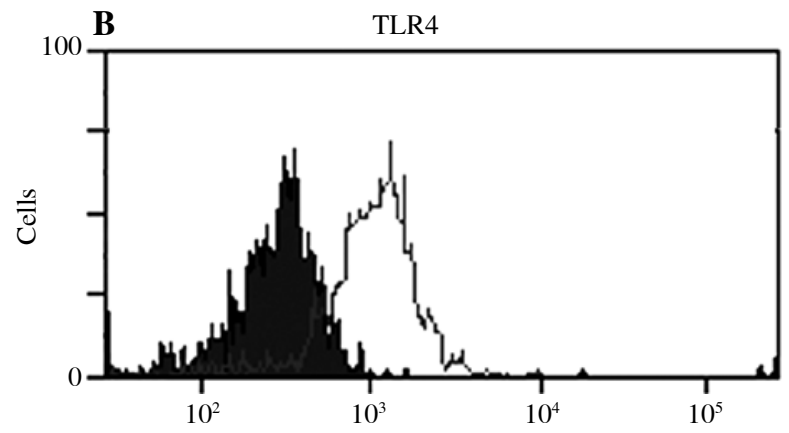

Fig. 2. Constitutive protein expression of surface TLRs in freshly isolated rat peritoneal mast cells. Representative flow cytometry histograms showing (A) TLR2, (B) TLR4, and (C) TLR5 protein expression. The shaded bars: isotype control and open bars: TLR expression 

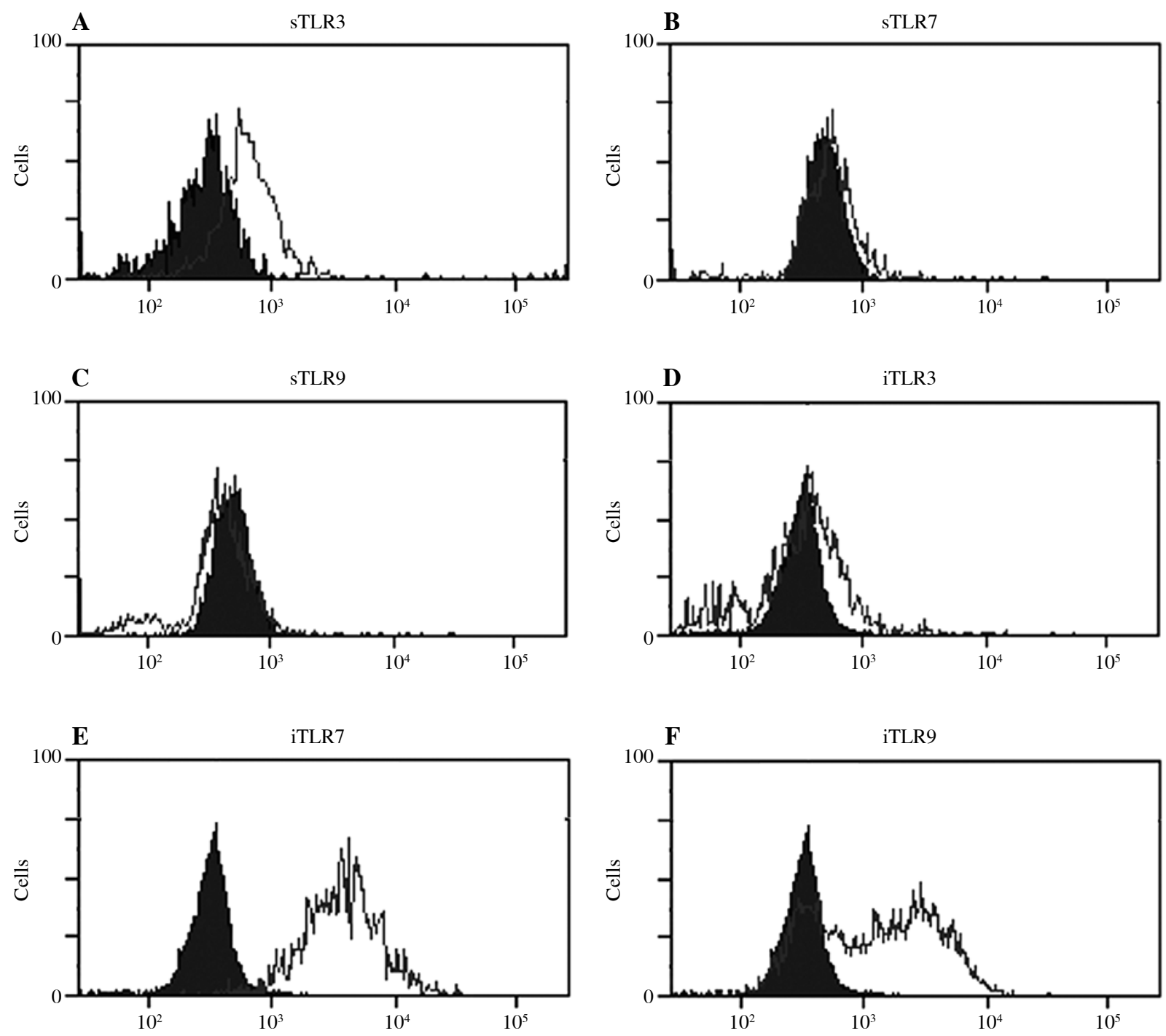

Fig. 3. Constitutive protein expression of both surface and intracellular TLRs in freshly isolated rat peritoneal mast cells. Representative flow cytometry histograms showing surface (A) sTLR3, (B) sTLR7, (C) sTLR9 and intracellular (D) iTLR3, (E) iTLR7, (F) iTLR9 protein expression. The shaded bars: isotype control and open bars: TLR expression

for other surface receptors. From among intracellular receptors expression of iTLR7 protein was on average two times higher than iTLR9 expression and more than three times higher than iTLR3 expression. We also noticed, that all receptors expressed both on the cell surface and intracellularly exhibit higher expression intracellularly than on the surface.

\section{Discussion}

Toll-like receptors constitute the most important class of PRRs. They are the major sensors of the innate immunity and are implicated in priming the adaptive immune response crucial for killing invading pathogens. Binding of TLR agonists to their receptors begins the activation of complex networks of intracellular signal transduction processes to coordinate the inflammation. TLR2 identifies and binds peptidoglycan, lipoteichoic acid, lipoproteins, lipoarabinomannan, and fungal zymosan. TLR4 is the receptor for lipopolysaccharide. It is worth pointing out that TLR2 and TLR4 recognize also some endogenous molecules that arise in the course of host response to infections, including high mobility group box 1 protein (HMGB1), heat shock proteins HSP60 and HSP70, $\beta$-defensins, and hyaluronan fragments. Bacterial flagellin is TLR5 agonist. TLR3, TLR7, and TLR8 recognize ligands of viral origin, i.e. double-stranded RNA (dsRNA) and single-stranded RNA (ssRNA). TLR9 senses unmethylated CpG sequenc- 
es in DNA molecules of viral or bacterial origin. TLR3, TLR7, and TLR9 molecules can also bind endogenous ligands, including endogenous nucleic acids (TLR3) and immune complexes containing nucleic acids (TLR7 and TLR9) [17-19].

Although the role of mast cells in antimicrobial host defence is well documented the expression of TLRs in these cells is not fully described. Furthermore, most studies on the TLR expression were conducted on mast cell lines or mast cell differentiated in vitro. It should be highlighted that these cells differ with respect to phenotype and activity from native tissue mast cells and can serve as replacements of mature mast cells to a limited degree only. TLR1 transcript was found in LAD [21] and KU812 [22] lines, as well as in cord blood-derived mast cells (CBMCs) [22] and human cultured mast cells (HCMCs) [23]. TLR2 and TLR4 mRNAs were described in LAD, HMC-1 [21, 24], KU812 [22], and MC/9 [25] cell lines, as well as in CBMCs [22, 26-28], mouse bone marrow-derived mast cells (BMMCs) [25, 29-33], and HCMCs [23]. TLR3 transcript was found in LAD, HMC-1, and P815 cell lines [23, 34] and in BMMCs [35] and HCMCs [23]. TLR5 transcript was described in LAD, HMC-1, and HCMCs [21, 23, 29, 36, 37], while TLR6 mRNA was found in MC/9 [25] and KU812 [22] lines, as well as in CBMCs [22, 26], BMMCs [25, 29-31, 33], and HCMCs [23]. It was also documented that LAD [23], HMC-1 [24], P815 cells [34], BMMCs [30], and HCMCs [24] express TLR7 and TLR9 mRNAs while TLR8 transcript was found in BMMCs [23]. Much less is known about the expression of TLR proteins in mast cells. It was shown that LAD and HMC-1 cells express TLR2, TLR4 [21, 24, 37], TLR5 [23, 36], TLR6, TLR7 [23], and TLR9 [21, 23, 37] proteins, while TLR1 protein was expressed only on LAD cells [21, 37]. The P815 cells express both TLR3 and TLR7 proteins [34]. HCMCs express TLR2, TLR6, TLR7, and TLR9 proteins [23], whilst CBMCs express TLR2 and TLR4 [26] and BMMCs express TLR3 [35] and TLR4 [30] proteins. Data documenting expression of TLRs in fully mature tissue mast cells are still far from sufficient and require further detailed study. The majority of TLR transcripts in matured mast cells were demonstrated in fetal skin-derived mast cells (FSMCs) and mast cells isolated from human lung and skin [26, 30]. Only TLR2, TLR3, and TLR4 proteins were found in mast cells from isolated human nasal polyps [22], murine peritoneal cavity [38], and FSMC [30]. Our previous studies have shown that freshly isolated rat peritoneal mast cells express both surface TLR2 and TLR4 molecules [20, 39], as well as TLR3 and TLR7 surface proteins [40, 41]. In this study we evaluated the expression of TLR receptors in native fully mature rat mast cells freshly isolated from the peritoneal cavity, that is connective tissue type mast cells. We found that these cells constitutively express mRNAs for TLR2, TLR3, TLR4, TLR5, TLR7, and TLR9. Our results from qRT-PCR analysis of TLR mRNA expression

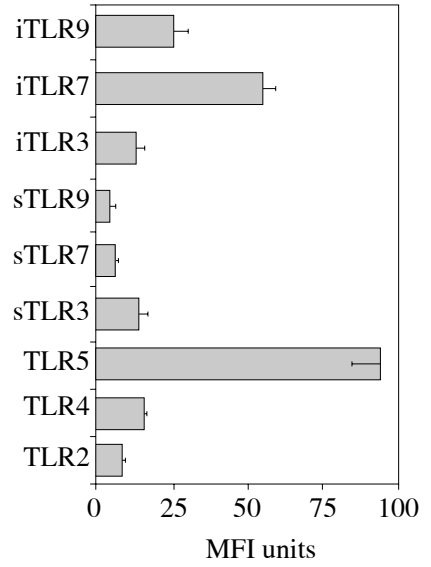

Fig. 4. Comparison of TLR expression at protein levels expressed as mean fluorescence intensity (MFI). Data are presented as the mean \pm SEM of four independent experiments

in rat peritoneal mast cells confirm and are coinciding with results of other authors who detected transcripts encoding TLR1, TLR2, TLR3, TLR4, TLR5, TLR6 and TLR8 in freshly isolated and purified mast cells [42]. Moreover, we have clearly documented that rat mast cells express TLR2, TLR4, and TLR5 on cell surface, while TLR3, TLR7, and TLR9 proteins are located both on the cell membrane and intracellularly. The highest expression was observed for TLR5 and the lowest for surface TLR7. These observations undoubtedly indicate that mature tissue mast cells have a broad set of TLR molecules, thus can recognize and bind bacterial, viral, and fungal PAMPs as well as various endogenous molecules generated in response to infection.

This work was supported by the Medical University of Łódź (Grant No. 503/6-164-01/503-61-001).

The authors declare no conflict of interest.

\section{References}

1. Wernersson S, Pejler G (2014): Mast cell secretory granules: armed for battle. Nat Rev Immunol 14: 478-494.

2. Krystel-Whittemore M, Dileepan KN, Wood JG (2016): Mast cell: a multi-functional master cell. Front Immunol 6: 620.

3. da Silva EZ, Jamur MC, Oliver C (2014): Mast cell function: a new vision of an old cell. J Histochem Cytochem 62: 698738.

4. Metz M, Grimbaldeston MA, Nakae S, et al. (2007): Mast cells in the promotion and limitation of chronic inflammation. Immunol Rev 217: 304-328.

5. Theoharides TC, Alysandratos KD, Angelidou A, et al. (2012): Mast cells and inflammation. Biochim Biophys Acta 1822: 21-33.

6. Shelburne CP, Abraham SN (2011): The mast cell in innate and adaptive immunity. Adv Exp Med Biol 716: 162-185. 
7. Modena BD, Dazy K, White AA (2016): Emerging concepts: mast cell involvement in allergic diseases. Transl Res 174: 98-121.

8. Trivedi NH, Guentzel MN, Rodriguez AR, et al. (2013): Mast cells: multitalented facilitators of protection against bacterial pathogens. Expert Rev Clin Immunol 9: 129-138.

9. Witczak P, Brzezińska-Błaszczyk E (2012): Mast cells in viral infections. Postepy Hig Med Dosw 66: 231-244.

10. Di Nardo A, Vitiello A, Gallo RL (2003): Cutting edge: mast cell antimicrobial activity is mediated by expression of cathelicidin antimicrobial peptide. J Immunol 170: 2274-2278.

11. Malaviya R, Twesten NJ, Ross EA, et al. (1996): Mast cells process bacterial Ags through a phagocytic route for class I MHC presentation to T cells. J Immunol 156: 1490-1496.

12. Malaviya R, Ross EA, MacGregor JI, et al. (1994): Mast cell phagocytosis of FimH-expressing enterobacteria. J Immunol 152: 1907-1914.

13. von Köckritz-Blickwede M, Goldmann O, Thulin P, et al. (2008): Phagocytosis-independent antimicrobial activity of mast cells by means of extracellular trap formation. Blood 111: 3070-3080.

14. Dimitriadou V, Mécheri S, Koutsilieris M, et al. (1998): Expression of functional major histocompatibility complex class II molecules on HMC-1 human mast cells. J Leukoc Biol 64: 791-799.

15. Kumagai Y, Takeuchi O, Akira S (2008): Pathogen recognition by innate receptors. J Infect Chemother 14: 86-92.

16. Takeuchi O, Akira $S$ (2010): Pattern recognition receptors and inflammation. Cell 140: 805-820.

17. Yamamoto M, Takeda K (2010): Current views of toll-like receptor signaling pathways. Gastroenterol Res Pract 2010: 240365.

18. Beutler BA (2009): TLRs and innate immunity. Blood 113: 1399-1407.

19. Kawasaki T, Kawai T (2014): Toll-like receptor signaling pathways. Front Immunol 5: 461.

20. Pietrzak A, Wierzbicki M, Wiktorska M, Brzezińska-Błaszczyk E (2011): Surface TLR2 and TLR4 expression on mature rat mast cells can be affected by some bacterial components and proinflammatory cytokines. Mediators Inflamm 2011: 427473.

21. Yoshioka M, Fukuishi N, Iriguchi S, et al. (2007): Lipoteichoic acid downregulates FceRI expression on human mast cells through Toll-like receptor 2. J Allergy Clin Immunol 120: 452-461.

22. McCurdy JD, Olynych TJ, Maher LH, Marshall JS (2003): Cutting edge: distinct Toll-like receptor 2 activators selectively induce different classes of mediator production from human mast cells. J Immunol 170: 1625-1629.

23. Kulka M, Alexopoulou L, Flavell RA, Metcalfe DD (2004): Activation of mast cells by double-stranded RNA: evidence for activation through Toll-like receptor 3. J Allergy Clin Immunol 114: 174-182.

24. Kubo Y, Fukuishi N, Yoshioka M, et al. (2007): Bacterial components regulate the expression of Toll-like receptor 4 on human mast cells. Inflamm Res 56: 70-75.

25. McCurdy JD, Lin TJ, Marshall JS (2001): Toll-like receptor 4-mediated activation of murine mast cells. J Leukoc Biol 70: 977-984.

26. Inomata N, Tomita H, Ikezawa Z, Saito H (2005): Differential gene expression profile between cord blood progenitor-derived and adult progenitor-derived human mast cells. Immunol Lett 98: 265-271.
27. Sundstrom JB, Little DM, Villinger F, et al. (2004): Signaling through Toll-like receptors triggers HIV-1 replication in latently infected mast cells. J Immunol 172: 4391-4401.

28. Varadaradjalou S, Féger F, Thieblemont N, et al. (2003): Tolllike receptor 2 (TLR2) and TLR4 differentially activate human mast cells. Eur J Immunol 33: 899-906.

29. Ikeda T, Funaba M (2003): Altered function of murine mast cells in response to lipopolysaccharide and peptidoglycan. Immunol Lett 88: 21-26.

30. Matsushima H, Yamada N, Matsue H, Shimada S (2004): TLR3-, TLR7-, and TLR9-mediated production of proinflammatory cytokines and chemokines from murine connective tissue type skin-derived mast cells but not from bone marrow-derived mast cells. J Immunol 173: 531-541.

31. Mrabet-Dahbi S, Metz M, Dudeck A, et al. (2009): Murine mast cells secrete a unique profile of cytokines and prostaglandins in response to distinct TLR2 ligands. Exp Dermatol 18: 437-444.

32. Stassen M, Müller C, Arnold M, et al. (2001): IL-9 and IL-13 production by activated mast cells is strongly enhanced in the presence of lipopolysaccharide: NF- $\mathrm{KB}$ is decisively involved in the expression of IL-9. J Immunol 166: 4391-4398.

33. Supajatura V, Ushio H, Nakao A, et al. (2001): Protective roles of mast cells against enterobacterial infection are mediated by Toll-like receptor 4. J Immunol 167: 2250-2256.

34. Yang H, Wei J, Zhang H, et al. (2009): Upregulation of Tolllike receptor (TLR) expression and release of cytokines from P815 mast cells by GM-CSF. BMC Cell Biol 10: 37.

35. Orinska Z, Bulanova E, Budagian V, et al. (2005): TLR3-induced activation of mast cells modulates $\mathrm{CD} 8+\mathrm{T}$-cell recruitment. Blood 106: 978-987.

36. Kulka M, Metcalfe DD (2006): TLR3 activation inhibits human mast cell attachment to fibronectin and vitronectin. Mol Immunol 43: 1579-1586.

37. Yoshioka M, Fukuishi N, Kubo Y, et al. (2008): Human cathelicidin CAP18/LL-37 changes mast cell function toward innate immunity. Biol Pharm Bull 31: 212-226.

38. Feng BS, He SH, Zheng PY, et al. (2007): Mast cells play a crucial role in Staphylococcus aureus peptidoglycan-induced diarrhea. Am J Pathol 171: 537-547.

39. Konopka Ł, Wierzbicki M, Brzezińska-Błaszczyk E (2010): Lipopolysaccharide from Porphyromonas gingivalis stimulates rat mast cells to cysteinyl leukotriene generation and upregulates Toll-like receptor- 2 and -4 expression. Int J Immunopathol Pharmacol 23: 803-810.

40. Witczak P, Pietrzak A, Wódz K, Brzezińska-Błaszczyk E (2014): Mast cells generate cysteinyl leukotrienes and interferon-beta as well as evince impaired IgE-dependent degranulation upon TLR7 engagement. Indian J Exp Biol 52: 589-596.

41. Witczak P, Pietrzak A, Słodka A, Brzezińska-Błaszczyk E (2013): Toll-like receptors 3 ligation directly and indirectly affects mast cell cysteinyl leukotriene generation. Cent Eur J Immunol 38: 343-348.

42. Passante E, Ehrhardt C, Sheridan H, Frankish N (2009): RBL$2 \mathrm{H} 3$ cells are an imprecise model for mast cell mediator release. Inflamm Res 58: 611-618. 